\title{
The Influence of Medial Collateral Ligament and Lateral Collateral Ligament Pie Crusting in Primary Total Knee Arthroplasty on Patient Reported Outcomes
}

\author{
Ethan Cornwell, ${ }^{1}$ Evan R. Deckard, ${ }^{2}$ Kevin A. Sonn, ${ }^{2}$ R. Michael Meneghini ${ }^{2}$ \\ ${ }^{1}$ Indiana University School of Medicine; ${ }^{2}$ Indiana University School of Medicine, Department of \\ Orthopaedic Surgery
}

\section{Background and Hypothesis:}

Pie-crusting of the collateral ligaments can help achieve balanced gaps in total knee arthroplasty (TKA) in knees with varus or valgus deformity. However, the effect of this technique on patient-reported outcome measures (PROMs) is unknown. The purpose of this study was to compare PROMs following primary TKA for patients with and without medial collateral ligament $(\mathrm{MCL})$ or lateral collateral ligament (LCL) pie-crusting.

\section{Experimental Design or Project Methods:}

We retrospectively reviewed 1,305 primary TKAs. Intraoperative MCL or LCL pie-crusting was documented in all operative reports and recorded. Prospectively collected preoperative, 4month postoperative, and minimum 1-year postoperative PROMs related to overall knee health, pain during functional activities, activity level, and overall satisfaction were compiled and compared between patients with and without MCL or LCL pie-crusting. Medians were evaluated with Kruskal-Wallis test adjusted for ties.

\section{Results:}

The cohort was $67 \%$ female with mean age 66 years and BMI $34.0 \mathrm{~kg} / \mathrm{m}^{2}$. MCL or LCL piecrusting was performed in $13.0 \%$ of the cohort. There were no intraoperative or postoperative ruptures of the MCL or LCL. $6.3 \%$ of conforming bearing TKAs required a collateral ligament pie crusting versus $21.5 \%$ of standard bearing TKA $(p<0.001)$. No significant differences were found in preoperative, 4-month, minimum 1-year, or change from preoperative baseline to minimum 1year PROMs with and without pie-crusting of the collateral ligaments ( $p \geq 0.095)$. However, the LCL pie-crusting group had slightly better PROMs at minimum 1-year.

\section{Conclusion and Potential Impact:}

These study results corroborate existing literature that pie-crusting of collateral ligaments is safe and effective to achieve a balanced TKA. Additionally, no significant outcome differences were found between groups with and without MCL or LCL pie-crusting. However, LCL pie-crusting for valgus knee correction had slightly better PROMs. Interestingly, conforming bearings may impart enhanced stability and mitigate the need for pie-crusting ligament releases in TKA. 